\title{
Effect of a Cosmetic Use with $2 \%$ Isostearyl-L-Ascorbic Acid Gel for Postinflammatory Hyperpigmentation and Postinflammatory Erythema in Acne Vulgaris
}

\author{
Ichiro Kurokawa ${ }^{1 *}$, Masako Watanabe ${ }^{2}$, Kazuhito Hayashibe ${ }^{3}$ \\ ${ }^{1}$ Department of Dermatology, Meiwa Hospital, Nishinomiya, Hyogo, Japan \\ ${ }^{2}$ Kobayashi Skin Clinic, Kobe, Japan \\ ${ }^{3}$ Hayashibe Skin Clinic, Sanda, Japan \\ Email: *kurokawa.i@meiwa-hospital.com
}

How to cite this paper: Kurokawa, I., Watanabe, M. and Hayashibe, K. (2020) Effect of a Cosmetic Use with $2 \%$ Isostearyl-L-Ascorbic Acid Gel for Postinflammatory Hyperpigmentation and Postinflammatory Erythema in Acne Vulgaris. Journal of Cosmetics, Dermatological Sciences and Applications, 10, 28-32.

https://doi.org/10.4236/jcdsa.2020.101004

Received: February 29, 2020

Accepted: March 20, 2020

Published: March 23, 2020

Copyright $\odot 2020$ by author(s) and Scientific Research Publishing Inc. This work is licensed under the Creative Commons Attribution International License (CC BY 4.0).

http://creativecommons.org/licenses/by/4.0/

\section{(c) (i) Open Access}

\begin{abstract}
Postinflammatory hyperpigmentation (PIH) and postinflammatory erythema (PIE) in acne vulgaris are important and refractory complications for patients with acne vulgaris. To clarify the effects of $2 \%$ isostearyl-L-ascorbic acid (ISAA) against PIH and PIE in acne vulgaris, a clinical pilot study with topical 2\% ISAA gel was performed in 25 acne patients with PIH and PIE. Topical ISAA gel was applied on the whole face with PIH and PIE in acne vulgaris twice a day for 3 months. Regarding PIH and PIE, investigator's global improvement rating (IGIR) was evaluated in 7-point scales according to the reduced area of PIH and PIE before and after the study. Remarkable improvement in PIH was observed in 7 patients $(28.0 \%)$ and in PIE in 12 (48\%) of the 25 patients. No adverse reactions were observed during the treatment. Topical ISAA application can be an alternative, non-invasive available treatment for PIH and PIE in acne vulgaris.
\end{abstract}

\section{Keywords}

Acne, Vitamin C, Postinflammatory Hyperpigmentation, Postinflammatory Erythema

In 2017, the new Japanese Dermatological Association (JDA) guideline for acne vulgaris was published [1]. Postinflammatory hyperpigmentation (PIH) and postinflammatory erythema (PIE) in acne vulgaris are important and refractory complications for patients with acne vulgaris. As one of the non-surgical treat- 
ments for PIH in acne vulgaris, chemical peeling with glycolic acid and subsequent iontophoresis with vitamins $\mathrm{C}$, vitamin $\mathrm{A}$, and vitamin $\mathrm{E}$ has been reported [2].

Vitamin $\mathrm{C}$ has multiple diversified effects such as anti-oxidation effects, inhibition of tyrosinase, proliferation of collagen synthesis and inhibition of metallo-matrix protease. Therefore, vitamin C can improve PIH and PIE in acne vulgaris.

Isostearyl-L-ascorbic acid (ISAA: Funcos C-IS), an amphipathic vitamin C derivative, has a distinct stable characteristic of highly transdermal absorption, allowing it to pass through the skin barrier and cell membrane inside keratinocytes and reaching into the dermis [3]. ISAA can increase collagen synthesis, inhibit matrix metalloprotease (MMP)-1, inhibit melanogenesis [4], and scavenge reactive oxygen species (ROS).

To clarify the effects of ISAA against PIH and PIE in acne vulgaris, a clinical pilot study with topical 2\% ISAA gel was performed in 25 acne patients with PIH and PIE. ISAA gel was supplied by Toyo Beauty Com Ltd', Osaka, Japan, containing $2 \%$ ascorbic acid, vitamin $\mathrm{A}$, and vitamin $\mathrm{E}$.

This study was approved by the Institutional Review Board of Meiwa Hospital (reference number: No. 28 - 05). 25 patients ( 2 men and 23 women) were enrolled in this study. Written informed consent was obtained from all patients. Mean age was 27.7 years ( 15 - 49 years). Severity of acne grade was mild to moderate. Concomitant medicines could be used during the study. Topical ISAA gel was applied on the whole face with PIH and PIE in acne vulgaris twice a day for 3 months. Regarding PIH and PIE, investigator's global improvement rating (IGIR) was evaluated on a 7-point scale from baseline: complete: $100 \%$, excellent: $75 \%$ - 99\%, marked: $50 \%$ - 75\%, moderate: $25 \%$ - 49\%, mild: $0 \%-25 \%$; no change: $0 \%$; worse in 1-month interval according to the reduced area of PIH and PIE before and after the study. During the treatment, adverse reactions were described.

The results including background of acne patients, IGIR in PIH and PIE, number of acne lesion and adverse reactions are summarized in Table 1. Remarkable (complete, excellent, and marked) improvement in PIH was observed in 7 patients (28.0\%) and in PIE in 12 (48\%) of the 25 patients. No adverse reactions were observed during the treatment. Representative cases were shown in Figure 1 (before and after the treatment).

We speculated that ISAA permeated into the hair follicle and inhibited melanogenesis, MMP-1 production, and inflammation by ROS and increased collagen synthesis, resulting in the improvement of PIH and PIE. Vitamin C also promotes wound healing and alters the inflammatory proliferative remodeling phase [5]. Topical vitamin $\mathrm{C}$ derivative was effective for perifollicular pigmentation [6]. Remarkable improvement with ISAA was observed in PIH and PIE in acne vulgaris without any adverse reactions in all patients during the treatment. ISAA gel contained vitamin E, which has an antioxidant effect and inhibits lipid 

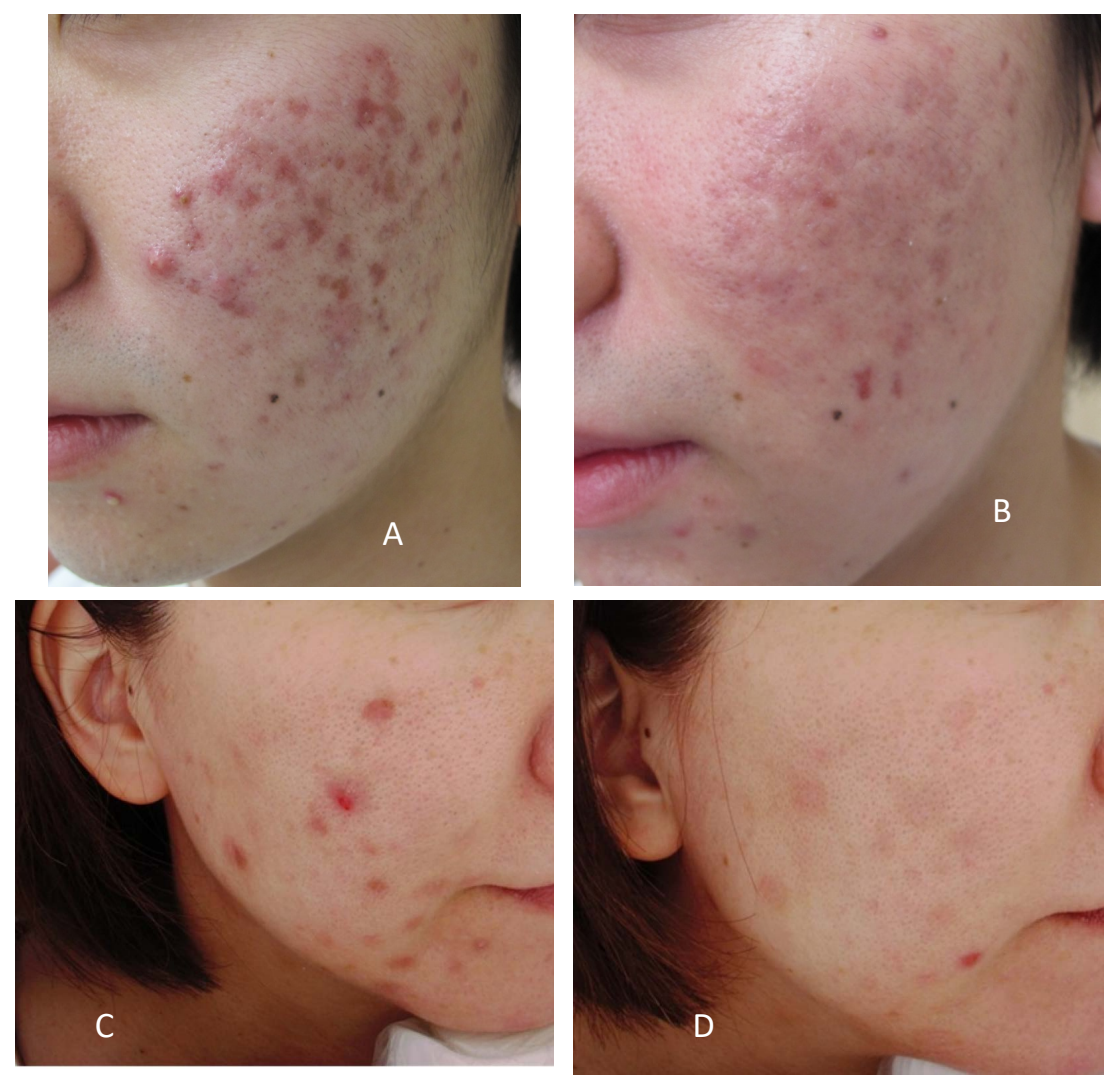

Figure 1. (A) Before the treatment, dense brown postinflammatory hyperpigmentation (PIH) and postinflammatory erythema (PIE) are observed on the left cheek. The patient had allergic contact dermatitis against adapalene and benzoyl peroxide. (B) After the 3-month treatment with topical ISAA, excellent improvement in PIH was observed. Marked improvement in PIE is observed. (C) Before the 3-month treatment with topical ISAA, dense brown PIH and PIE are observed. (D) After the 3-month treatment with topical ISAA, excellent improvement was observed in PIH and PIE.

Table 1. Summary of background of acne patients (gender, age, duration of suffering, concomitant drugs), Investigator's global improvement rating (IGIR) in postinflammatory hyperpigmentation (PIH) and postinflammatory erythema (PIE), number of acne lesions on the whole face (inflammatory lesions and comedo), and adverse reaction.

\begin{tabular}{|c|c|c|c|c|c|c|c|c|c|c|c|}
\hline \multirow[t]{2}{*}{ case } & \multirow[t]{2}{*}{ gender } & \multirow[t]{2}{*}{ age } & \multirow{2}{*}{$\begin{array}{l}\text { duration of } \\
\text { suffering }\end{array}$} & \multirow[t]{2}{*}{ concomitant drugs } & \multirow[t]{2}{*}{ PIH(IGIR) } & \multirow[t]{2}{*}{ PIE(IGIR) } & \multicolumn{2}{|c|}{$\begin{array}{c}\text { number of } \\
\text { inflammatory lesions }\end{array}$} & \multicolumn{2}{|c|}{$\begin{array}{l}\text { number of } \\
\text { comedo }\end{array}$} & \multirow[t]{2}{*}{$\begin{array}{l}\text { adverse } \\
\text { reactions }\end{array}$} \\
\hline & & & & & & & $0 \mathrm{w}$ & $12 \mathrm{w}$ & $0 \mathrm{w}$ & $12 \mathrm{w}$ & \\
\hline 1 & $\mathrm{~F}$ & 42 & 27 & $\mathrm{BPO} / \mathrm{ADA}$ & marked & moderate & 6 & 0 & 9 & 2 & none \\
\hline 2 & $\mathrm{~F}$ & 25 & 13 & $\mathrm{BPO} / \mathrm{ADA}$ & moderate & mild & 15 & 20 & 33 & 30 & none \\
\hline 3 & M & 22 & 8 & $\begin{array}{c}\text { Seijobofuto, heparinoid, } \\
\text { BPO/ADA }\end{array}$ & moderate & excellent & 2 & 0 & 14 & 14 & none \\
\hline 4 & $\mathrm{~F}$ & 15 & 5 & heparinoid, BPO/ADA & marked & complete & 18 & 3 & 20 & 8 & none \\
\hline 5 & $\mathrm{~F}$ & 39 & 19 & $\mathrm{BPO} / \mathrm{ADA}$ & worse & moderate & 5 & 1 & 12 & 12 & none \\
\hline 6 & $\mathrm{~F}$ & 24 & unknown & $\mathrm{BPO} / \mathrm{ADA}$ & excellent & mild & 24 & 5 & 40 & 13 & none \\
\hline 7 & $\mathrm{~F}$ & 47 & 34 & BPO & mild & complete & 2 & 0 & 5 & 4 & none \\
\hline 8 & $\mathrm{~F}$ & 40 & unknown & heparinoid, $\mathrm{BPO} / \mathrm{ADA}$ & mild & marked & 5 & 0 & 7 & 2 & none \\
\hline
\end{tabular}




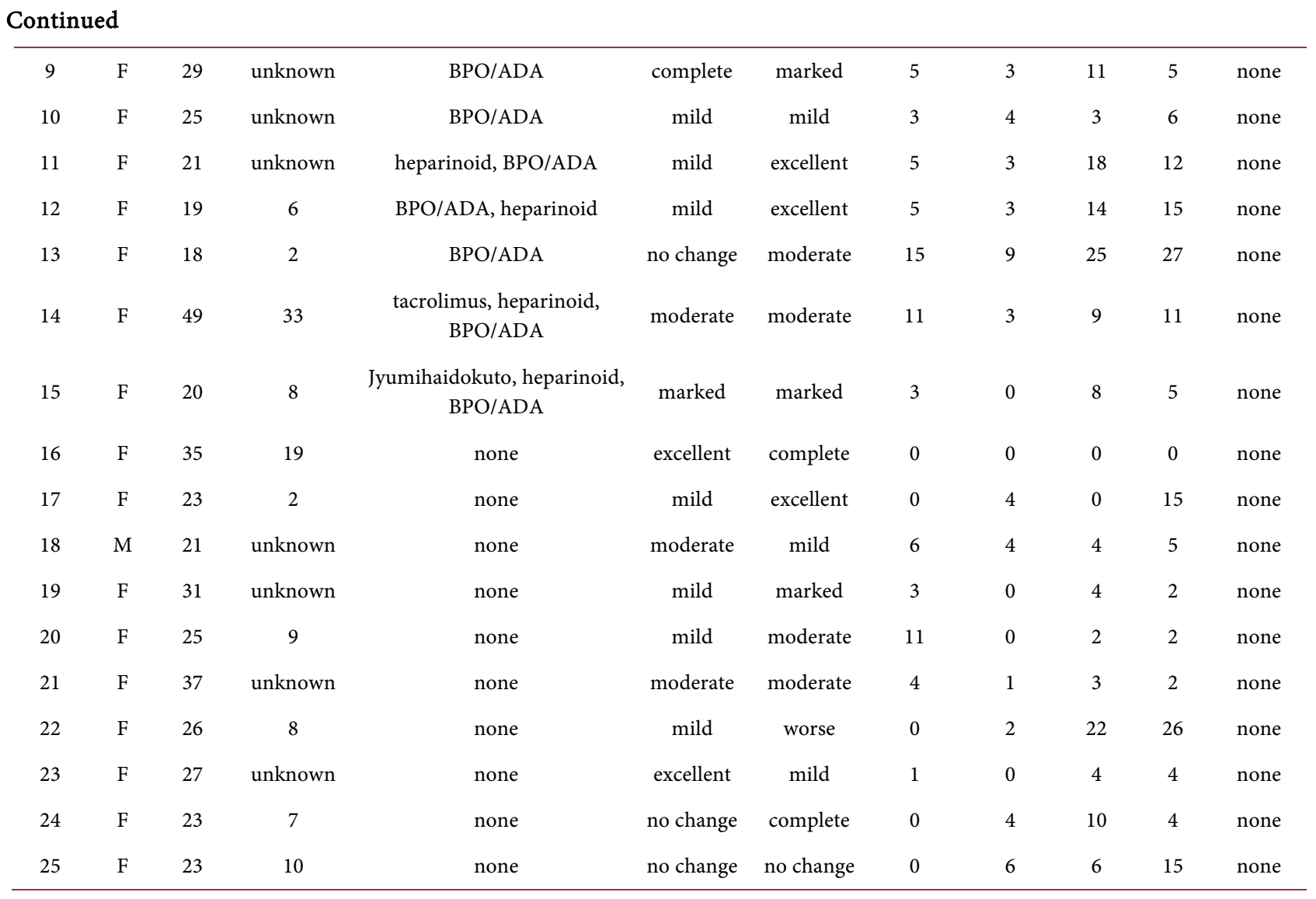

BPO: benzoyl peroxide; CLDM: clindamaycin; ADA: adapalen.

peroxide, and vitamin A, which inhibits abnormal hyperkeratinization in follicular infundibulum. Topical ISAA application can be an alternative, non-invasive available treatment for PIH and PIE in acne vulgaris.

In the future, a long-term, half-sided face study is necessary to evaluate the effect of ISAA against PIH and PIE in acne vulgaris.

\section{Funding Acknowledgement}

This research received no specific grant from any funding agency in the public, commercial, or not-for-profit sectors.

\section{Conflicts of Interest}

The authors declare no conflict of interest in preparing this article.

\section{References}

[1] Hayashi, N., Akamatsu, H., Iwatsuki, K., et al. (2018) Japanese Dermatological Association Guidelines: Guidelines for the Treatment of Acne Vulgaris 2017. The Journal of Dermatology, 45, 898-935. https://doi.org/10.1111/1346-8138.14355

[2] Kurokawa, I., Oiso, N. and Kawada, A. (2017) Adjuvant Alternative Treatment with Chemical Peeling and Subsequent Iontophoresis for Postinflammatory Hyperpigmentation, Erosion with Inflamed Red Papules and Non-Inflamed Atrophic Scars in 
Acne Vulgaris. The Journal of Dermatology, 44, 401-405.

https://doi.org/10.1111/1346-8138.13634

[3] Shibayama, H., Hisama, M., Matsuda, S. and Ohtsuki, M. (2008) Permeation and Metabolism of a Novel Ascorbic Acid Derivative, Disodium Isostearyl 2-L-Ascorbyl Phosphate, in Human Living Skin Equivalent Model. Skin Pharmacology and Physiology, 21, 235-248. https://doi.org/10.1159/000139128

[4] Matsuda, S., Shibayama, H., Hisama, M., Ohtsuki, M. and Iwaki, M. (2008) Inhibitory Effects of a Novel Ascorbic Derivative, Disodium Isostearyl 2-O-L-Ascorbyl Phosphate on Melanogenesis. Chemical and Pharmaceutical Bulletin, 56, 292-297. https://doi.org/10.1248/cpb.56.292

[5] Mohammed, B.M., Fisher, B.J., Kraskauskas, D., et al. (2016) Vitamin C Promotes Wound Healing through Novel Pleiotropic Mechanisms. International Wound Journal., 13, 572-584. https://doi.org/10.1111/iwj.12484

[6] Inui, S. and Itami, S. (2007) Perifollicular Pigmentation Is the First Target for Topical Vitamin C Derivative Ascorbyl 2-Phosphate 6-Palmitate (APPS): Randomized, Single-Blinded, Placebo-Controlled Study. The Journal of Dermatology, 34, 221-223. https://doi.org/10.1111/j.1346-8138.2007.00256.x 